\title{
Nonlinear Granger causality and its application in the analysis of epileptic EEG and ECG signal
}

\author{
Peng $\mathrm{Du}^{1, \mathrm{a}^{*}}$, Jia-Fei Dai ${ }^{2}$, Jin $\mathrm{Li}^{3}$ and Qianli Mal ${ }^{4}$ \\ ${ }^{1}$ Image Processing and Image Communications Key Lab. Nanjing Univ. of Posts \& Telecomm. , \\ Nanjing 210003, China \\ ${ }^{2}$ Institute of Biomedical Electronic Engineering, School of Electronic Science and Engineering, \\ Nanjing University, Nanjing, 210002, China \\ ${ }^{3}$ College of Physics and Information Technology, Shaanxi Normal University - Xi'an 710062, China \\ ${ }^{a}$ E-mail: wangj@njupt.edu.cn, ${ }^{b}$ E-mail: houfz@cpu.edu.cn
}

\begin{abstract}
Keywords: nolinear Granger causality, epileptic, kernel function
Abstract. In this paper, a method based on the nolinear Granger causality is used to analyze epilptic EEG and ECG signal. Polynomial kernel function, Gaussian kernel function and sigmoid kernel function are used to map the linear data in low dimensional input space into high dimensional feature space .In this space linear Granger method can be used to analyse the biomedical signals. The results show that the effect of ECG signals to EEG signals is more significant than that of EEG signals to ECG signals and the result by normal subjects is more significant than that of epileptic subjects. This study is helpful for the analysis of epileptic patient's EEG and ECG signal..
\end{abstract}

\section{Introduction}

Epilepsy is a common brain disease, which is harmful to health. It is caused by transient central nervous system dysfunction. Brain electrical signal contains a lot of physiological and pathological information. It plays a very important role in clinical medicine and brain science [1].In epileptic seizures, brain waves are often accompanied by high amplitude slow wave, spike wave, sharp wave. The EEG reflects the computer activity and functional status of brain tissue [2]. The change of ECG signal in the regulation of autonomic nerve and the evaluation of autonomic nerve function which has better specificity and sensitivity than other physiological parameters [3].

The purpose of this paper is to analyze the causal relationship of the epileptic EEG and ECG signal. Time series can make the study of complex engineering system more easily [4]. A method is proposed by Granger, in the linear regression model, if the prediction error of the first sequence is reduced after the introduction of second sequences, it can be said that the second sequence has a causal effect on the first sequence [5]. This theory in economics, meteorology has extensive application. The significance of Granger's causal relationship is to reflect the amount of transfer information between the two subsystems, so the introduction of kernel method to the nonlinear field of Granger causality is very meaningful.

In this paper, the nonlinear Granger causality index is used to analyze the physiological signals of epilepsy and to discuss the relationship between EEG and ECG. Kernel function theory is a method to realize the support vector method under the condition of linear non separable data. So the inner product of feature space can be represented by a kernel function, the essence of kernel function is corresponding to the inner product of the high dimensional space. Therefore, kernel function results in the formation of the high dimensional space of feature mapping.

\section{Nonlinear Granger causality}

The given training set $\left\{\varphi_{n}, \omega_{n}\right\} \quad(n=1,2, . ., N+m)$,to meet the Markov process, namely $X_{i}=\left(\varphi_{i}, \ldots, \quad \varphi_{i+m-1}\right)^{\top}, Y_{i}=\left(\omega_{i}, \ldots, \omega_{i+m-1}\right), x_{i}=\varphi_{i}(i=1, \ldots, N)$. For $\alpha \in\{1, \ldots, m\}$, 
the $\alpha$ th samples of $X$ form vector $\mu_{\alpha} \in \Re^{N}$,for without loss of generality, the mean value of $\mu_{\alpha}$ is 0 . General $x=\left(x_{1}, \ldots, x_{N}\right)^{\top}$ normalized treatment, the mean value is also taken 0 . The sample collection $\mu_{1}, \ldots, \quad \mu_{m}$ extended linear space $H$. The $N \times N$ matrix $X$ of the expansion of the space is consistent with $K=X^{\top} X[6]$, take $v_{1}, \ldots v_{m}$ as a set of standard orthogonal vectors of the matrix $K$ with nonzero eigenvalues. The projection operator for linear space $\mathrm{H}$ is defined as

$$
P=\sum_{i=1}^{m} v_{i} v_{i}^{\top}
$$

Here, $x=P x, x^{\tilde{x}}$ is the projection of $x$ on $H[8]$. Thus, for a given $X$, prediction error is $\varepsilon_{\mathrm{x}}=\|\mathrm{x}-\mathrm{x}\|^{2}=1-\mathrm{x}^{\mathrm{t}} \mathrm{x}$.

Using both $\mathrm{X}$ and $\mathrm{Y}$, the $\mu_{1}, \ldots, \quad \mu_{\mathrm{m}}$ and $\mathrm{Y}$ sample collection of $\gamma_{1}, \ldots, \gamma_{\mathrm{m}}$ form the linear space $\mathrm{H}^{\prime} \subseteq \mathfrak{R}^{\mathrm{N}}$. The $\mathrm{Z}$ is $2 \mathrm{~m} \times \mathrm{N}$ matrix with $\mu_{\alpha}, \gamma_{\alpha}$ as column vectors. $\mathrm{H}^{\prime}$ is the range of the matrix $K=Z^{\top} Z$ [6]. Calculated according to the formula (1) $\mathrm{H}^{\prime}$, the projection operator $\mathrm{P}^{\prime}$, linear regression values of the vector $x^{\prime}=P^{\prime} x$, predicted error $\varepsilon_{x y}=\left\|x-x^{\prime}\right\|^{2}=1-x^{\prime \top} x^{\prime}$. Linear Granger causality index is:

$$
\delta(Y \rightarrow X)=\frac{\varepsilon_{x}-\varepsilon_{x y}}{\varepsilon_{x}}=\frac{X^{\prime \top} X^{\prime}-X^{\top} X}{1-X^{\prime \top} X^{\prime}}
$$

By means of a feature map, the kernel function method can map the linear data in low dimensional input space into high dimensional feature space (Hilbert space) [9]. The kernel method is used for nonlinear mapping, which makes use of high dimensional space to deal with data [7].

Kernel function can be expressed as $K\left(X, X^{\prime}\right)=\sum_{\alpha} \lambda_{\alpha} \Psi_{\alpha}(X) \Psi_{\alpha}\left(X^{\prime}\right)$ and its the sign function $\sqrt{\lambda_{\alpha}} \Psi_{\alpha}$, the eigenfunction expansion of linear space in mind is $\mathrm{H}, \mathrm{N} \times \mathrm{N}$ inner product matrix $K_{i j}=k\left(X_{i}, X_{j}\right), H$ and $N \times N$ matrix $K$ space expansion consistent [6]. Similar to the linear Granger causality, by calculating the linear space $\mathrm{H}$, the projection operator $\mathrm{P}$ can be obtained for prediction error $\varepsilon_{\mathrm{x}}$ while $\mathrm{X}$ is given.

$\mathrm{Y}$ Series is introduced according to the $\mathrm{X}$ series and $\mathrm{Y}$ Series generation $\mathrm{Z}$ sequence, compute the $\mathrm{N} \times \mathrm{N}$ inner product matrix $\mathrm{K}^{\prime}, \mathrm{K}_{\mathrm{ij}}{ }^{\prime}=\mathrm{k}\left(\mathrm{Z}_{\mathrm{i}}, \mathrm{Z}_{\mathrm{j}}\right), \mathrm{H}^{\prime}$ is the range of the matrix $\mathrm{K}^{\prime}$ [6]. Similar to the linear Granger causality, by calculating the linear space $\mathrm{H}^{\prime}$, the projection operator $\mathrm{P}^{\prime}$ can obtain the prediction error for a given XY. According to (2) formula, Granger's causal coefficient can be obtained.

This paper will discuss the common three kinds of kernel functions: $p$ order polynomial kernel function $k_{p}\left(X, X^{\prime}\right)=\left(1+X^{\top} X^{\prime}\right)^{p}$, the parameter is $p ;$ Gaussian kernel function $k_{\sigma}\left(X, X^{\prime}\right)=\exp \left(-\frac{\left(X-X^{\prime}\right)^{\top}\left(X-X^{\prime}\right)}{2 \sigma^{2}}\right)$, the parameter $\sigma$ reflect the complexity and sigmoid kernel function $K_{\alpha \beta}\left(X, X^{\prime}\right)=\tanh \left(\alpha X^{\top} X^{\prime}+\beta\right)$, the parameters are $\alpha$ and $\beta$ [9].

\section{Epileptic EEG and ECG signal and nonlinear Granger causality analysis}

EEG and ECG data recording. The EEG data is from the latest clinical collection, including epileptic and normal EEG signal which were collected from the General Hospital of the Nanjing Military Region. 
The EEG and ECG recording were collected by using the standard methodology (10-20 system) and 17 electrode cap , which includes FP1, FP2, F3, F4, C3, C4, P3, P4, O1, O2, F7, F8, T3, T4, T5, T6,ECG. The original EEG and ECG signals sample frequency is 512HZ.

Granger causality of the normal sample EEG signal and ECG signal. By calculating the Granger causality between the normal subjects and the EEG signals, we can obtain the causality diagram of the EEG and ECG signals. The results of the three kinds of kernel function are shown in Fig. 1.

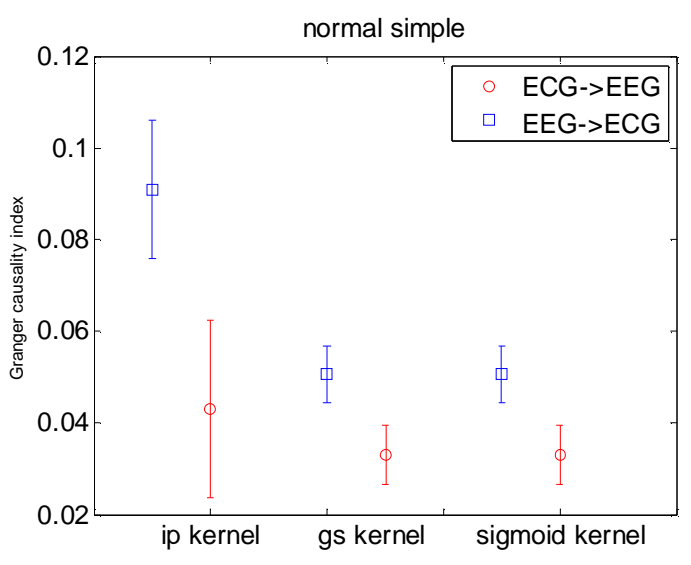

Fig. 1 The error bar of the normal sample Granger causal index

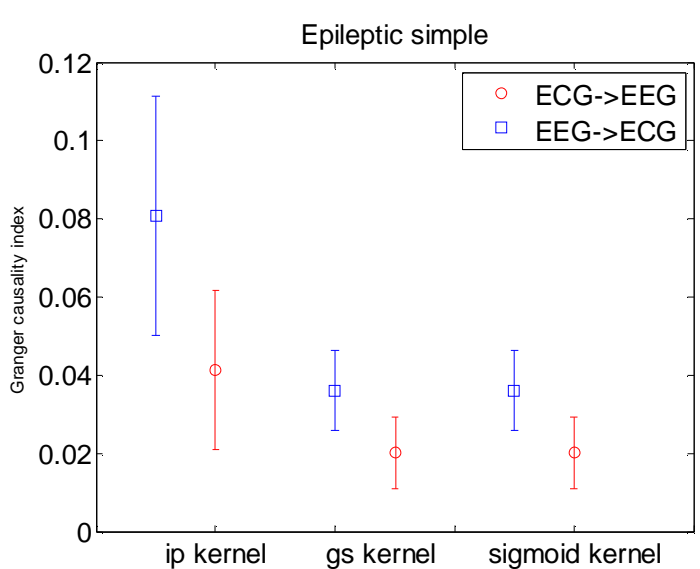

Fig. 2 The error bar of the epileptic sample Granger causal index

With independent $t$ test for sample mean by SPSS, the significance of the polynomial kernel function is 0.001 , the sigmoid kernel function is 0.001 , and the Gaussian kernel function is 0.001 , the significance of this three are all less than 0.005 .So, the $t$ test can be obtained by the confidence degree of 0.95 . Three kinds of kernel functions can be significantly improved.

It can be found that the EEG signal of the normal person has a significant effect on the heart electrical signal, and the effect of the ECG signal to EEG signal is small. By comparing the effect of three kinds of kernel functions, we can find that the significant level of the polynomial kernel function is more obvious while using suitable parameter and the significant level of Gaussian kernel and sigmoid kernel are close to each other. When the parameter changes the result of Gaussian kernel and sigmoid kernel have little change.

Analysis of Granger causality of the epileptic sample EEG signal and ECG signal. By calculating the Granger causality between the epileptic subjects and the EEG signals, we can obtain the causality diagram of the EEG and ECG signals. The results of the three kinds of kernel functions are shown in Fig. 2.

With independent $t$ test for sample mean by SPSS, the significance of the polynomial kernel function is 0.035 , the sigmoid kernel function is 0.044 , and the Gauss kernel function is 0.045 , the significance of this three are all less than 0.005 .So, the $t$ test can be obtained by the confidence degree of 0.95 .Three kinds of kernel functions can be significantly improved. By comparing the effect of three kinds of kernel functions, we can find that the significant level of the polynomial kernel function is more obvious while using suitable parameter and the significant level of Gaussian kernel and sigmoid kernel are close to each other. When the parameter changes the result of Gaussian kernel and sigmoid kernel have little change.

It can be found that the EEG signal of the normal person has a significant effect on the heart electrical signal, and the effect of the ECG signal to EEG signal is small. By comparing EEG signal and ECG signal of normal subjects and patients with Epilepsy. It can be found that the normal simple EEG's effect to ECG signal is more obvious despite epileptic simple can also tell the different direction of effect by the confidence degree of 0.95 . 


\section{Conclusions}

This paper analyzes the EEG signal and ECG signal based on the nonlinear Granger causality. Polynomial kernel function, Gaussian kernel function and sigmoid kernel function are used to extend the linear Granger causality to nonlinear space.

Through the analysis of the experimental results, it is found that there is a significant causal relationship between ECG signal and EEG signal. The effect of EEG on the signal of ECG is more significant than that of the signal to the brain. In addition, compared with the normal data analysis results, the effect of EEG signal to the EEG signal is weak. This indicates that the ECG signal of epileptic patients is related to the signal of the brain electrical signal, and the factors that need to be considered when the signal is analyzed. This is associated with a high amplitude slow wave, spike wave, sharp wave and so on. Therefore, it is essential to take into account the influence factors of EEG signal in the analysis of epileptic patient's ECG signal.

\section{Acknowledgements}

Project supported by the National Natural Science Foundation of China (Grant Nos. 61271082, 61201029, 61401518), Jiangsu Provincial Key R \& D Program (Social Development) (Grant No. BE2015700), the Natural Science Foundation of Jiangsu Province (Grant No. BK20141432), the Foundation of Nanjing General Hospital of Nanjing Military Command (Grant No. 2014019) and the Fundamental Research Funds for the Central Universities (Grant No. FY2014LX0039).

\section{References}

[1] R.S. Fisher, W.V.E. Boas, W. Blume, C. Elger, P. Genton, P. Lee, J. E. Jr, Epileptic seizures and epilepsy: definitions proposed by the International League Against Epilepsy (ILAE) and the International Bureau for Epilepsy (IBE), Epilepsia. 46 (2005) 470-472.

[2] I. Mporas, V.Tsirka, E.I. Zacharaki, M.Koutroumanidis, M.Richardson, V.Megalooikonomou, Seizure detection using EEG and ECG signals for computer-based monitoring, analysis and management of epileptic patients, Expert Systems with Applications. 42 (2015) 3227-3233

[3] J. Nawrath, M.C. Romano, M. Thiel, I.Z. Kiss, M. Wickramasinghe, J. Timmer, J. Kurths, B. Schelter, Distinguishing Direct from Indirect Interactions in Oscillatory Networks with Multiple Time Scales, Phys. Rev. Lett. 104 (2010) 338-346.

[4] L. Lu, X. Xia, Anti-synchronization of nonlinear-coupled spatiotemporal chaotic systems, Acta Physica Sinica. 58 (2009) 814-818.

[5] C.W. J. Granger, GRANGER C W J, Investigating Causal Relations by Econometric Models and Cross-spectral Meth, Econometrica. 37 (1969): 424-438.

[6] B. Schoelkopf, K. Muan, K. Fukumizu, Computing functions of random variables via reproducing kernel Hilbert space representations[J]. Statistics and Computing. 25 (2015): 755-766.

[7] D. Marinazzo, M. Pellicoro,S. Stramaglia, Kernel Granger causality and the analysis of dynamicalnetworks. Physical Review. E. 66 (2008) 056215.

[8] D. Marinazzo, M. Pellicoro,S. Stramaglia, Kernel method for nonlinear granger causality. Phys Rev Lett. 100 (2008) 144103.

[9] G. Camps, J.D. Martın, J.L. Rojo, E. Soria, Fuzzy sigmoid kernel for support vector classifiers. Neurocomputing. 62 (2004) 501-506. 